\title{
Symptomatic and Asymptomatic Chronic Carotid Artery Occlusion on High-Resolution MR Vessel Wall Imaging
}

\author{
(D) M. Wan, (D) Yan, (D). Xu, (D) Z. Hou, (D). Kang, (D). Cui, (D). Yu, (D). Song, (D).K. Hui, (D) Y. Wang,
} (D) Z. Miao, (D) X. Lou, and (D) N. Ma

\begin{abstract}
BACKGROUND AND PURPOSE: Chronic carotid artery occlusion remains a poorly understood risk factor for subsequent stroke, and potential revascularization is dependent on understanding the anatomy and nature of the occlusion. Luminal imaging cannot assess the nature of an occlusion, so the internal structure of the occlusion must be inferred. The present study examines the signal characteristics of symptomatic and asymptomatic carotid occlusion that may point to management differentiation.

MATERIALS AND METHODS: We prospectively recruited patients who were diagnosed with chronic carotid artery occlusion defined as longer than 4 weeks and confirmed by DSA. All patients underwent high-resolution MR vessel wall imaging examinations after enrollment. Baseline characteristics, vessel wall imaging features, and DSA features were collected and evaluated. The vessel wall imaging features included segment involvement, signal intensity, contrast enhancement, and vessel wall thickness. The symptomatic and asymptomatic chronic carotid artery occlusions were compared.
\end{abstract}

RESULTS: A total of 44 patients with 48 lesions were included in this study from February 2020 to December 2020 . Of the 48 lesions, 35 (72.9\%) were symptomatic and 13 (27.1\%) were asymptomatic. There was no difference in baseline and DSA features. On vessel wall imaging, $\mathrm{Cl}$ and $\mathrm{C} 2$ were the most commonly involved segments $(91.7 \%$ and $68.8 \%$, respectively). Compared with symptomatic lesions, asymptomatic lesions were more often isointense $(69.2 \%)$ in the distal segment $(P=.03)$. Both groups had diffuse wall thickening (80\% and $100 \%)$.

CONCLUSIONS: Signal characteristics between those with symptomatic and asymptomatic carotid artery occlusions differ in a statistically significant fashion, indicating a different structure of the occlusion.

ABBREVIATIONS: $C C A O=$ chronic carotid artery occlusion; $\mathrm{VWI}=$ vessel wall imaging

hronic carotid artery occlusion (CCAO) accounts for $6.5 \%$ of ischemic strokes. ${ }^{1}$ CCAO may present as an incidental finding in asymptomatic patients, or it may present clinically as a TIA or a severe disabling stroke. The annual risk of TIA or ischemic stroke of asymptomatic CCAO is estimated to be between $2 \%$ and $8 \% .^{2-4}$ The risk of stroke recurrence in symptomatic CCAO is $8 \%$ at 30 days, $10 \%$ at 1 year, and $14 \%$ at 5 years. ${ }^{5}$

Received May 31, 2021; accepted after revision September 28.

From the Department of Interventional Neuroradiology (M.W., L.Y., Z.H., R.C., Y.Y., J.S., Z.M., N.M.) and Neurology (K.K., Y.W.), Beijing Tiantan Hospital, Capital Medical University, Beijing, China; China National Clinical Research Center for Neurological Diseases (M.W., L.Y., Z.H., K.K., R.C., Y.Y., J.S., Y.W., Z.M., N.M.), Beijing, China; Department of Neurology (Z.X.), The First Affiliated Hospital of College of Medicine, Zhejiang University, Hangzhou, China; Department of Radiology (X.L.), Chinese PLA General Hospital, Beijing, China; Department of Neurology (M.W.), Shijingshan Teaching Hospital of Capital Medical University, Beijing Shijingshan Hospital, Beijing, China; and Department of Radiology and Radiological Sciences (F.K.H.), Johns Hopkins Hospital, Baltimore, Maryland.

This work was supported by Beijing Yangfan Plan, XMLX201844, National Natural Science Foundation of China, 81730048, 81825012.
CCAO may cause ischemic stroke due to an embolism from the occluded segment, compromised hemodynamics, and a mixture of both embolic and hemodynamic factors. ${ }^{6}$ The role of hemodynamics in patients with symptomatic and asymptomatic CCAO has been demonstrated in previous studies. ${ }^{4,7}$ The biology and architecture of the occluded segment of a CCAO remain unclear. Conventional imaging modalities including CTA, MRA, ultrasonography, and DSA can evaluate the structural and hemodynamic functional information of nonoccluded vessels but cannot provide sufficient information about the features of vessel segments with no substantial flow. High-resolution MR vessel

Min Wan, Long Yan, and Ziqi Xu contributed equally to this article.

Please address correspondence to Ning Ma, MD, Department of Interventional Neuroradiology, China National Clinical Research Center for Neurological Diseases, Beijing Tiantan Hospital, Capital Medical University, No. 119 South 4th Ring West Rd, Fengtai District, Beijing 100070, China; e-mail: maning_03@hotmail.com

- Indicates open access to non-subscribers at www.ajnr.org

Indicates article with online supplemental data.

http://dx.doi.org/10.3174/ajnr.A7365 
wall imaging (VWI) has been widely used in atherosclerotic diseases of the carotid and intracranial arteries. ${ }^{8-11}$ VWI can determine plaque size, intraplaque hemorrhage, large lipid core, and intraluminal thrombus and can evaluate dissection flaps and subintimal plaque. ${ }^{12}$ In addition, 3D VWI with variable flip angles can offer imaging with improved longitudinal coverage in a shorter imaging time. ${ }^{13,14}$ VWI has been reported to be practicable and accurate in the diagnosis of CCAO. ${ }^{15,16}$ VWI for CCAO can provide more information and direct visualization of the occluded segment, such as segment involvement, signal intensity, enhancement characteristics, and vessel wall thickness. However, few studies have focused on the characteristics of the occluded segment of CCAO on VWI. ${ }^{17}$ Therefore, we conducted this study to assess the differing imaging characteristics between symptomatic and asymptomatic CCAO.

\section{MATERIALS AND METHODS Study Population}

This was a prospective study, approved by the institutional review board of the hospital. Informed consent was obtained from all patients or their representatives before enrollment. Patients admitted to the Department of Interventional Neuroradiology of Beijing Tiantan Hospital and the Department of Neurology of The First Affiliated Hospital of Zhejiang University who had been diagnosed with carotid artery occlusion by sonography, CTA, or MRA were selected. Carotid artery occlusion by sonography, CTA, or MRA was defined as $100 \%$ cross-sectional truncation of the vessel lumen. "Chronic" was defined as the patient having a carotid occlusion diagnosis for $>4$ weeks, confirmed by DSA, with TICI grade $0 .{ }^{18,19}$ All patients underwent DSA and VWI examinations after enrollment. The symptomatic CCAO group comprised patients who were admitted due to anterior ipsilateral ischemic events in the territory of the occluded vessel. The asymptomatic CCAO group was patients diagnosed with carotid artery occlusion incidentally during the course of routine clinical care. Ischemic events included ischemic stroke and TIA on the ipsilateral hemisphere or retinal artery. The exclusion criteria were the following: 1) contraindications to VWI or DSA examination; 2) near-occlusion or pseudo-occlusion confirmed by DSA; 3) intracranial chronic carotid artery occlusion; and 4) cardioembolic risk factors.

\section{Data Collection}

Baseline data including sex, age, body mass index, atherosclerotic risk factors, antithrombotic agents taken before and after diagnosis of CCAO, history of radiation, and time of the ischemic event onset were collected. Risk factors were defined as the following: hypertension defined as a history of hypertension or the patient taking any hypotensive drugs; diabetes mellitus defined as a history of diabetes mellitus, the patient taking any hypoglycemic agents, or a glycosylated hemoglobin level of $\geq 7 \%$; hyperlipidemia defined as a history of hyperlipidemia or receiving lipid-lowering treatment; and coronary artery disease defined as a history of myocardial infarction or angina pectoris. Smoking was defined as current smokers or a smoking history. VWI and DSA features were recorded. On VWI, features of segment involvement, signal intensity of the occluded segment, contrast enhancement, and vessel wall thickness were evaluated. On DSA, the imaging features of the vascular stump and the level of the distal reconstitution were also evaluated.

\section{Vessel Wall Imaging}

VWI was performed on a 3T Magnetom Trio MR imaging scanner (Siemens) or a 3T Discovery MR750 scanner (GE Healthcare) with a head/neck coil. The 3D T1-weighted turbo spin-echo sequences on the Magnetom Trio MR imaging scanner were obtained with the following parameters: $\mathrm{TR} / \mathrm{TE}=760 / 15 \mathrm{~ms}$, section partial Fourier factor $=0.75$, turbo factor $=60$, echo spacing $=4.52 \mathrm{~ms}$, parallel imaging acceleration $=3$. The FOV was $240 \times 220 \times 172 \mathrm{~mm}$ (Foot-Head $\times$ Anterior-Posterior $\times$ Right-Left), and the voxel size was $0.54 \times 0.54 \times 0.54 \mathrm{~mm}$. The 3D T1-weighted turbo spin-echo sequences on the Discovery MR750 were obtained with the following parameters: $\mathrm{TR} / \mathrm{TE}=675 / 21 \mathrm{~ms}$, echo-train length $=24$, echo spacing $=7.5 \mathrm{~ms}$, bandwidth $=62.50 \mathrm{kHz}$, phase and HyperSense acceleration (GE Healthcare) $=2$, FOV $=180 \times 180 \mathrm{~mm}$ (Superior-Inferior $\times$ Right-Left). The pixel size was $0.6 \times 0.6 \mathrm{~mm}$, and the section thickness was $0.8 \mathrm{~mm}$. Other MR imaging scans included 3D TOF MRA, T2-weighted imaging, and contrastenhanced T1-weighted imaging. Images were reconstructed using the Reformate tool in the Advantage Workstation 4.5 (GE Healthcare) or the D multiple planer reconstruction tool in the Siemens workstation.

\section{DSA Imaging}

All DSA studies were performed by 2 experienced interventional neuroradiologists with $>10$ years' experience each. During angiography, the bilateral common carotid arteries and bilateral vertebral arteries received contrast injections in the anteriorposterior and lateral projections, respectively.

\section{Imaging Analysis}

All MR images were reviewed separately by a neuroradiologist with 6 years' experience and a neurologist with 8 years' experience who were blinded to clinical and medical information. A third reader was used to resolve disagreements in assessing the segment involvement. We divided each ICA into 7 segments (C1-C7) according to the classification of Bouthillier et al. ${ }^{20}$ To measure signal intensities, we placed ROIs at the occluded segment on the coronal T1weighted turbo spin-echo sequences, with maximal size and adjacent sternomastoid muscle by the consensus decision of the 2 investigators. Signal intensity was categorized as hypointensity (less than the signal intensity of the adjacent sternocleidomastoid muscle), isointensity (equal to that of the adjacent sternocleidomastoid muscle), hyperintensity (more than that of the adjacent sternocleidomastoid muscle), and heterogeneous intensity (a mix of the above).

The occluded segment was divided into the origin segment (origin of the ICA) and the distal segment (beyond the origin of the ICA) to assess the signal intensity separately. Contrast enhancement was determined by comparing pre- and postcontrast T1-weighted images focused on the intraluminal thrombus. Vessel wall thickness was evaluated by T1-weighted turbo spinecho sequences in the petrous ICA. The petrous ICA was divided into proximal, middle, and distal segments for measuring vessel wall thickness. Diffuse wall thickening was defined as a mean wall thickness of $>1.5 \mathrm{~mm}^{21}$ Time intervals between the last 

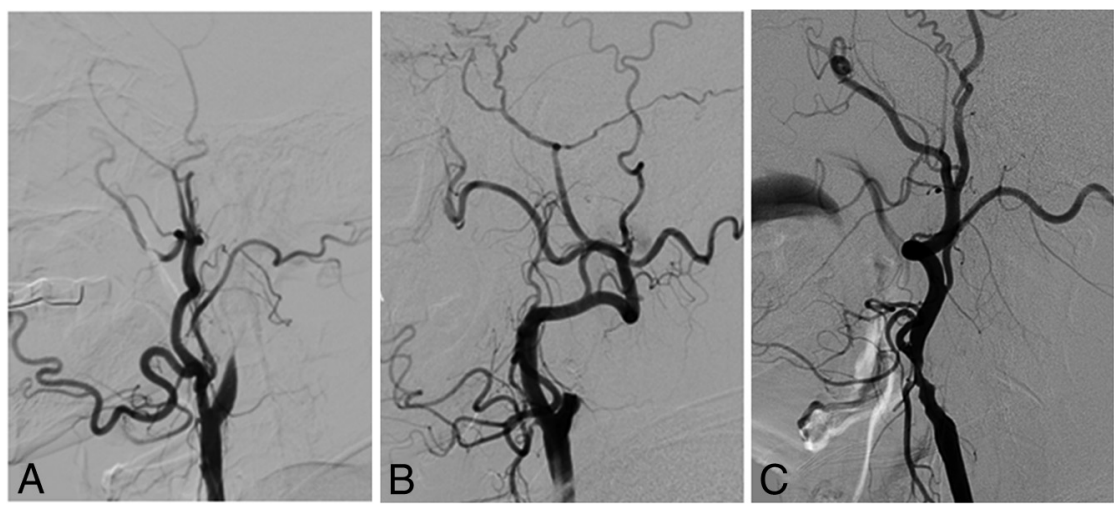

FIG 1. Stump condition: tapered stump (A), blunt stump (B), no stump (C)

Table 1: Baseline data of patients with asymptomatic and symptomatic CCAO

\begin{tabular}{lccr}
\hline & $\begin{array}{c}\text { Asymptomatic } \\
(\boldsymbol{n}=9)\end{array}$ & $\begin{array}{c}\text { Symptomatic } \\
(\boldsymbol{n}=35)\end{array}$ & $\begin{array}{c}\boldsymbol{P} \\
\text { Value }\end{array}$ \\
\hline Male (No.) (\%) & $8(88.9)$ & $34(97.1)$ & .37 \\
Age (mean) (yr) & $59(\mathrm{SD}, 13.35)$ & $60.51(\mathrm{SD}, 11.46)$ & .73 \\
Body mass index (mean) & $25.30(\mathrm{SD}, 3.49)$ & $24.40(\mathrm{SD}, 3.12)$ & .46 \\
Hypertension (No.) (\%) & $7(77.8)$ & $26(74.3)$ & $>.99$ \\
Diabetes mellitus (No.) (\%) & $3(33.3)$ & $12(34.3)$ & $>.99$ \\
Hyperlipidemia (No.) (\%) & $7(77.8)$ & $28(80.0)$ & $>.99$ \\
Coronary artery disease (No.) (\%) & $1(11.1)$ & $3(8.6)$ & $>.99$ \\
Smoker (No.) (\%) & $4(44.4)$ & $15(42.9)$ & $>.99$ \\
Antithrombotic treatment before diagnosis of & $1(11.1)$ & $8(22.9)$ & .75 \\
$\quad$ CCAO (No.) (\%) & $3(33.3)$ & $18(51.4)$ & .55 \\
Antithrombotic treatment after diagnosis of & & & \\
$\quad$ CCAO (No.) (\%) & $0(0.0)$ & $1(2.9)$ & $>.99$ \\
History of radiation (No.) (\%) & $1(1,2.5)$ & $2(1,4)$ & .74 \\
Time intervals between DSA and VWI (median) & & & \\
$\quad$ (IQR) (days) & & & \\
\hline
\end{tabular}

Note:-IQR indicates interquartile range.

ischemic event onset to VWI were divided into 4 stages ( $<7$ days, 7-30 days, 30-90 days, and $\geq 90$ days). The difference in signal intensity across the 4 time points was evaluated.

DSA images were reviewed by the 2 operators. According to the contrast filling within the ICA from the common carotid artery and the shape, the morphology of the carotid stump was categorized as tapered, blunt, and no stump (Fig 1). The level of the distal collateral reconstitution was defined as the most proximal site visible at the distal end of the occlusion, divided into proximal or distal cavernous segments.

\section{Statistical Analysis}

We performed statistical analysis using SPSS software, Version 20.0 (IBM). Normal distribution measurement data were expressed as mean (SD), while skew distribution data were expressed as median and interquartile range. Counting data were expressed in frequencies and percentages. We compared baseline data of symptomatic and asymptomatic patients. DSA features and VWI features were compared between those with symptomatic and asymptomatic CCAO. The Student $t$ test (normal distribution) or Mann-Whitney $U$ test (skewed distribution) and the $\chi^{2}$ test or Fisher exact test were used according to the situation. $P<.05$ was considered a statistically significant difference.

\section{RESULTS \\ Patient Characteristics}

Overall, a total of 44 patients with 48 lesions were included in this study from February 2020 to December 2020: 22 (50.0\%) in Beijing Tiantan Hospital and $22(50.0 \%)$ in The First Affiliated Hospital of Zhejiang University. Of the 44 patients, 35 (79.5\%) were symptomatic and 9 (20.5\%) were asymptomatic. In symptomatic patients, 32 patients had ischemic stroke (29 ipsilateral hemispheres and 3 retinal arteries) and 3 patients had experienced TIA (1 ipsilateral hemisphere and 2 retinal arteries). One (2.0\%) patient had a history of radiation. Baseline characteristics are shown in Table 1; there was no difference in baseline characteristics between symptomatic and asymptomatic patients. The time intervals between DSA and VWI were 1 day (interquartile range, 1-2.5 days) in the asymptomatic group and 2 days (interquartile range, 1-4 days) in the symptomatic group $(P=.74)$.

\section{Comparison between Symptomatic and Asymptomatic CCAO}

DSA showed no difference in stump morphology and the level of distal collateral reconstitution between the 2 groups (Online Supplemental Data).

On VWI, C1 and C2 were the most commonly involved segments in both symptomatic CCAO (97.1\% and 68.6\%) and asymptomatic CCAO (76.9\% and $69.2 \%$ ). Two patterns of signal intensity were detected in the origin segment (isointensity and heterogeneous intensity). There were 7 (20.0\%) instances of isointensity and 27 (77.1\%) of heterogeneous intensity in symptomatic CCAO (Fig 2). There were $5(38.5 \%)$ instances of isointensity and $5(38.5 \%)$ of heterogeneous intensity in those with asymptomatic CCAO $(P=.15)$. Three patterns of signal intensity were detected in the distal segment (hyperintensity, isointensity, and heterogeneous intensity). There was a higher prevalence of isointensity (69.2\%) in the asymptomatic CCAO compared with the symptomatic CCAO $(P=.03)$ group. There were 14 patients (15 lesions) with contrast-enhanced scans. Nine scans in 13 patients with symptomatic CCAO were demonstrated to be contrast-enhanced, as well as 2 scans in 2 patients with asymptomatic CCAO $(P=.95)$. Both patients with symptomatic, and asymptomatic CCAO had a high prevalence of diffuse thickening of the vessel wall ( $80 \%$ and $100 \%$ respectively).

\section{Difference in Signal Intensity at Different Stages of Symptomatic CCAO}

In symptomatic CCAO, both the origin segment and distal segment showed no difference at the following 4 stages: $<7$ days, 730 days, 30-90 days, and $\geq 90$ days (Tables 2 and 3). 

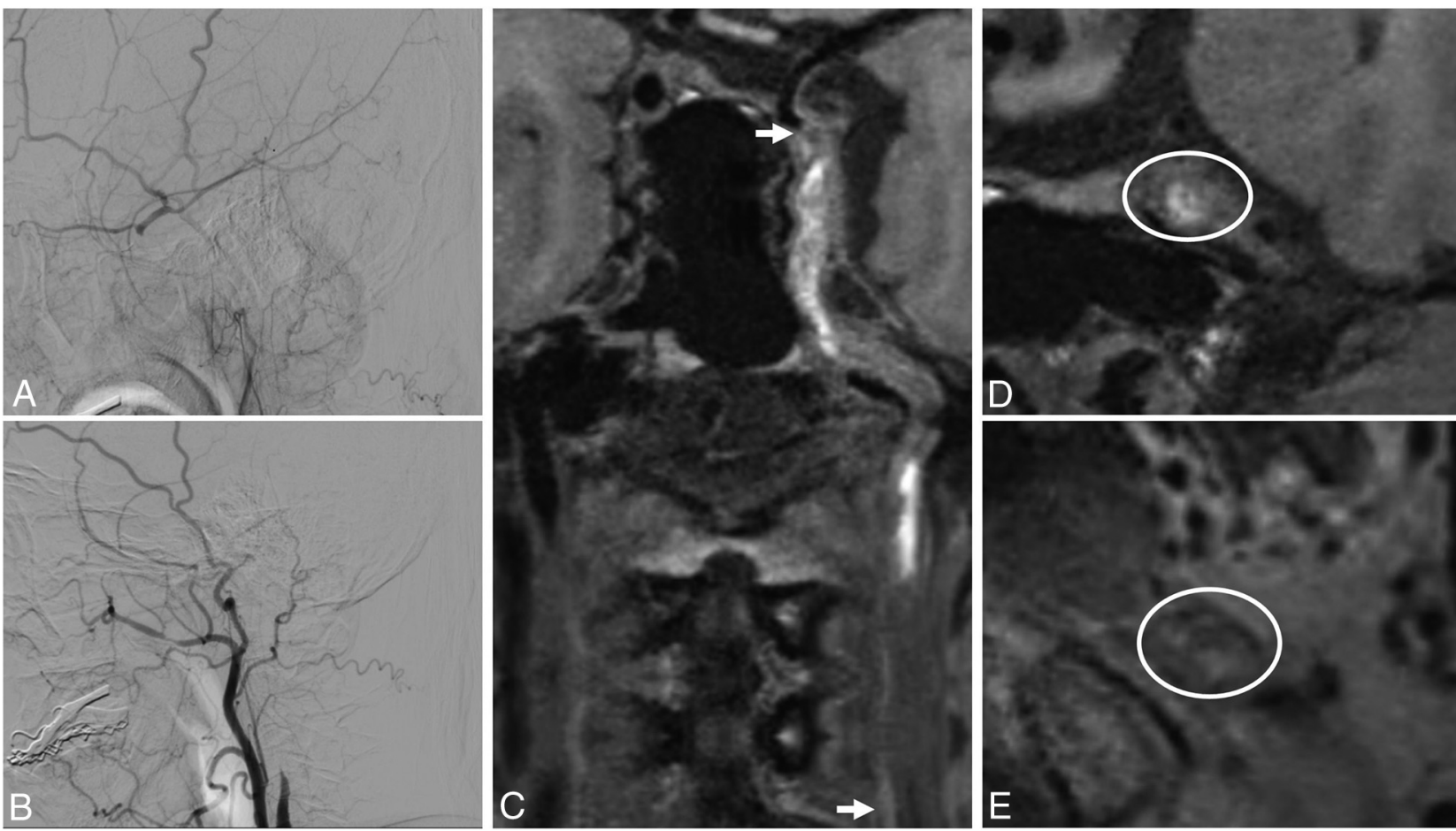

FIG 2. An adult man who presented with blurred vision for 1 month and right-limb weakness for 10 days. On DSA, the occluded segment is from $C 1$ (B) to C6 (A). On VWI, 3D T7-weighted turbo spin-echo sequences show that the occluded segment is Cl (arrow) to C6 (arrow) (C). The signal intensity of the distal segment in a elliptical region-of-interest (ROI) (D), and the signal intensity of the origin segment is heterogeneous intensity in a elliptical ROI (E).

Table 2: Difference in signal intensity at the origin segment of symptomatic CCAO at different stages

\begin{tabular}{cccccc}
\hline & $\begin{array}{c}\text { Hyperintensity } \\
(\boldsymbol{n}=0)\end{array}$ & $\begin{array}{c}\text { Isointensity } \\
(\boldsymbol{n}=7)\end{array}$ & $\begin{array}{c}\text { Hypointensity } \\
(\boldsymbol{n}=0)\end{array}$ & $\begin{array}{c}\text { Heterogeneous } \\
\text { Intensity }(\boldsymbol{n}=27)\end{array}$ & $\begin{array}{c}\boldsymbol{P} \\
\text { Value }\end{array}$ \\
\hline $\begin{array}{c}<\text { days } \\
(\text { No.) }(\%)\end{array}$ & $0(0.0)$ & $0(0.0)$ & $0(0.0)$ & $5(14.7)$ & .69 \\
$\begin{array}{c}7-30 \text { days } \\
(\text { No. })(\%)\end{array}$ & $0(0.0)$ & $4(57.1)$ & $0(0.0)$ & $16(47.1)$ & \\
$\begin{array}{c}30-90 \text { days } \\
(\text { No. })(\%)\end{array}$ & $0(0.0)$ & $2(28.6)$ & $0(0.0)$ & $10(29.4)$ & \\
$\geq 90$ days & $0(0.0)$ & $1(14.3)$ & $0(0.0)$ & $3(8.8)$ & \\
$($ No.) $(\%)$ & & & & & \\
\hline
\end{tabular}

Table 3: Difference in signal intensity at the distal segment of symptomatic CCAO at different stages

\begin{tabular}{cccccc}
\hline & $\begin{array}{c}\text { Hyperintensity } \\
(\boldsymbol{n}=9)\end{array}$ & $\begin{array}{c}\text { Isointensity } \\
(\boldsymbol{n}=14)\end{array}$ & $\begin{array}{c}\text { Hypointensity } \\
(\boldsymbol{n}=0)\end{array}$ & $\begin{array}{c}\text { Heterogeneous } \\
\text { Intensity }(\boldsymbol{n}=12)\end{array}$ & $\begin{array}{c}\boldsymbol{P} \\
\text { Value }\end{array}$ \\
\hline $\begin{array}{c}<7 \text { days } \\
(\text { No.) }(\%)\end{array}$ & $2(22.2)$ & $1(7.1)$ & $0(0.0)$ & $2(16.7)$ & .29 \\
$\begin{array}{c}7-30 \text { days } \\
(\text { No. })(\%)\end{array}$ & $2(22.2)$ & $6(42.9)$ & $0(0.0)$ & $8(67.7)$ & \\
$\begin{array}{c}30-90 \text { days } \\
(\text { No. })(\%)\end{array}$ & $4(44.4)$ & $4(28.6)$ & $0(0.0)$ & $2(16.7)$ & \\
$\geq 90$ days & $1(11.1)$ & $3(21.4)$ & $0(0.0)$ & $0(0.0)$ & \\
$($ No.) $(\%)$ & & & & &
\end{tabular}

\section{Comparison of DSA with VWI}

The accuracy of VWI for the diagnosis of total CCAO was $100 \%$. The level of occlusion differed between DSA and VWI, with a consistency rate of $27.1 \%$. Most of the lesions were shown to be shorter on VWI than DSA, and a tiny lumen could be seen beyond the occluded segment on VWI but was considered occluded on DSA (Fig 3).

\section{DISCUSSION}

The present study demonstrates that asymptomatic CCAO lesions have a higher prevalence of isointensity compared with symptomatic CCAO lesions. The most common occluded segments in CCAO lesions were $\mathrm{C} 1$ and $\mathrm{C} 2$. Most of the CCAO lesions had diffuse thickening of the vessel wall on VWI, so VWI may provide detailed characterization of the occlusion for patients with CCAO.

VWI for the diagnosis of CCAO has been reported to have high accuracy and sensitivity using DSA as a reference. $^{15,22,23}$ A few studies have focused on the signal intensity of the occluded segment of the CCAO. Previous studies have reported a higher prevalence of heterogeneous intensity at the beginning of the occluded segment and a $47.5 \%$ presence of high signal intensity in the occluded segment. ${ }^{15,17}$ Atherosclerosis is the major cause (about 70\%) of CCAO, and most lesions are located at the origin of the ICA. ${ }^{24}$ The deterioration of the atherosclerotic plaque 


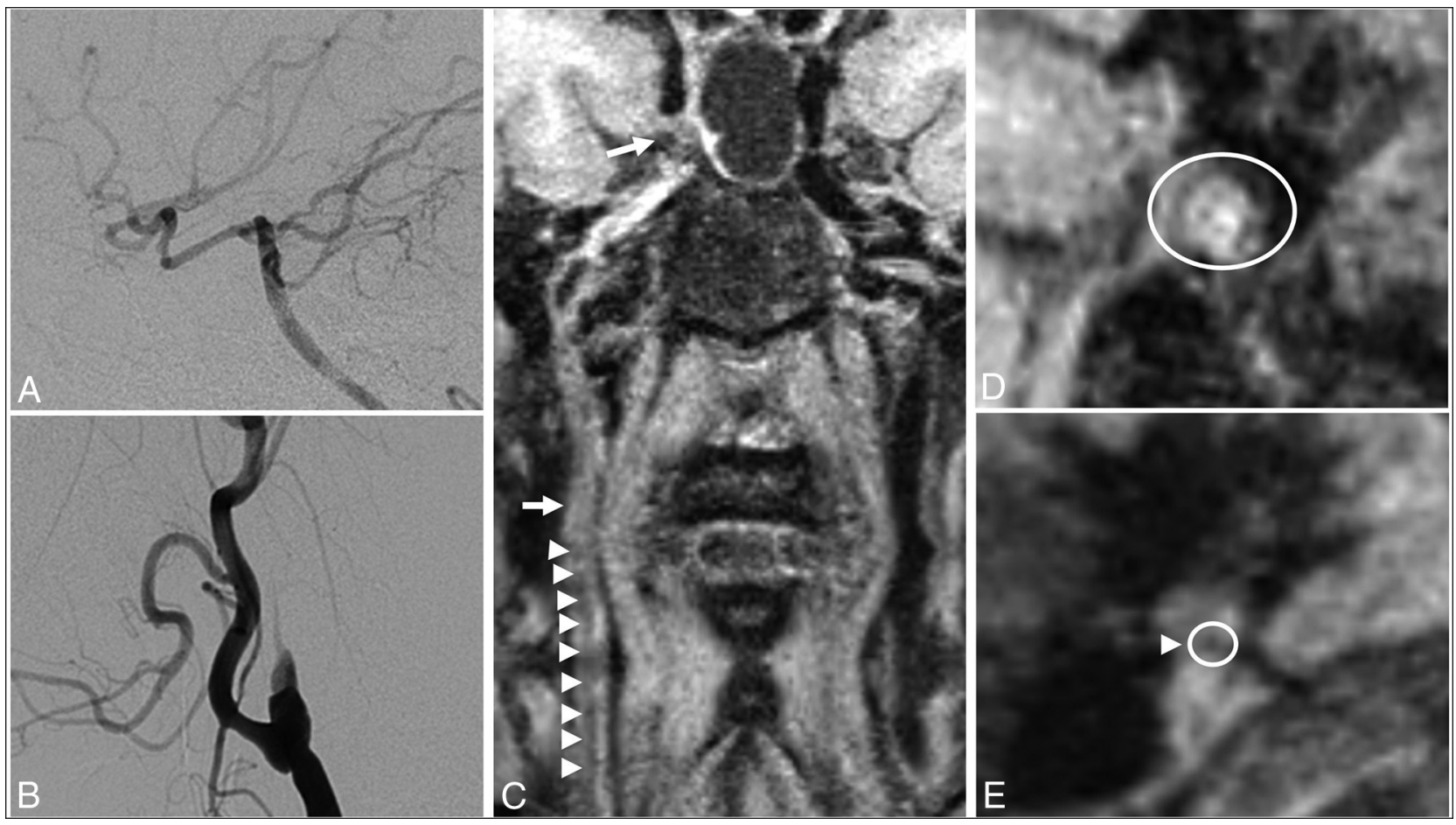

FIG 3. An adult woman who presented with dizziness for 2 months. On DSA, the occluded segment is from $\mathrm{Cl}(B)$ to $\mathrm{C7}(A)$. On VWI, 3D T7weighted turbo spin-echo sequences show that the occluded segment was C2 (arrow) to C4 (arrow), and Cl did not have total occlusion (arrowheads, C). The signal intensity of distal segment is isointensity in a elliptical region-of-interest (ROI) (D). The origin segment showed a tiny lumen (arrowhead), in a circular ROI which was considered an occluded segment on DSA (E).

leads to occlusion of the carotid artery and formation of the intraluminal thrombus within the distal segment. The signal intensities of the origin and distal segments are usually different. It is difficult to describe the whole occluded segment with uniform signal intensity. In this study, we divided the occluded segment into 2 parts to evaluate the signal intensity: the origin segment (the origin of the ICA) and the distal segment (distal C1 and above). Heterogeneous signal intensity was frequently encountered in the origin segment, but there was no difference between the 2 groups. The most common signal intensity in the distal segment was isointensity in asymptomatic lesions, which was not the case in symptomatic lesions $(P=.03)$.

Cerebral hemorrhage can be chronologically divided into different stages by MR imaging: hyperacute, acute, early subacute, late subacute, and chronic stage. ${ }^{25,26}$ Intraplaque hemorrhage of atherosclerosis on high-resolution MR imaging is also chronologically divided into fresh, recent, and old stages. ${ }^{27} \mathrm{We}$ proposed that the signal intensity of the distal segment may vary with time, similar to the signal intensity of cerebral hemorrhage and intraplaque hemorrhage. We selected different time intervals from the last onset of symptoms to the VWI examination in reference to cerebral hemorrhage and intraplaque hemorrhage. However, there was no difference in signal intensity at different time points. The same phenomenon has been observed in studies about intracranial largevessel arterial occlusion. ${ }^{28}$ The reason may be that the exact time of the CCAO is uncertain and there is spontaneous recanalization and reformation of the thrombus in the occluded segment.

In this study, all the lesions in the asymptomatic group and $80 \%$ in the symptomatic group had a diffuse thickening of the vessel wall. In a previous study, diffuse wall thickening in the petrous ICA was correlated with proximal ICA steno-occlusive disease. $^{29}$ With the increasing degree of stenosis, diffuse wall thickening became severe (present in $1.4 \%, 5.3 \%, 5.9 \%$, and $80.4 \%$ of ipsilateral proximal ICAs, respectively, in patients with stenosis of $1 \%-49 \%, 50 \%-69 \%, 70 \%-99 \%$, and total occlusion, respectively). ${ }^{29}$ The probable cause is edema of the vessel wall due to long-term ischemia. Nutrients of the intima and inner media are supplied by diffusion from the lumen, and the adventitia is supplied by the vasa vasorum. ${ }^{30}$ The vasa vasorum often arose directly from the lumen of the carotid artery and the arterial branching points. ${ }^{31}$ When the carotid artery was occluded, the vessel wall lost its nutrient supply and edema occurred.

DSA remains the criterion standard for the diagnosis of vascular disease; it can evaluate structural and hemodynamic functional information but cannot provide information on the vessel wall. VWI has been widely used in atherosclerosis of the carotid artery for its sufficient black-blood effects, excellent scan efficiency, high isotropic resolution and signal-to-noise ratio, and large spatial coverage. ${ }^{32,33}$ Compared with DSA, VWI can provide more information about the occluded segment. In this study, $72.9 \%$ of occluded segments detected by VWI were shorter than those detected by DSA. A previous study also found a similar result. ${ }^{16}$ The tiny lumen can be observed beyond the occluded segment on VWI, but it was considered occluded on DSA. We hypothesize that this was a pseudo-occlusion caused by collapse of the vessel wall due to inadequate compensatory proximal and distal blood flow. Endovascular interventional therapy has become a major treatment for CCAO. The stump condition and 
distal ICA reconstitution on DSA are important factors affecting successful recanalization. ${ }^{34}$ It is necessary to identify the true occluded segment, and VWI may provide more information to help improve the success rate of recanalization. ${ }^{16}$

There were some limitations of this study. First, the MR imaging scanner was different in the 2 centers. Second, 3D T1weighted turbo spin-echo sequences were used to evaluate occluded segments without additional flow suppression, possibly affecting the evaluation of slow blood flow in the proximal-to-distal occluded segments. Third, 9 patients included in the asymptomatic group were detected occasionally, so selection bias may exist. Due to the small sample size, the findings in the present study need to be interpreted with caution. Fourth, only a portion of patients underwent enhanced scans, possibly not fully demonstrating the characteristics of the occluded segment. Finally, the conclusion of this study needs to be confirmed by a larger amount of data research in the future.

\section{CONCLUSIONS}

VWI can provide further insight into the occluded segment of CCAO. The signal intensity was demonstrated to be different between symptomatic and asymptomatic CCAO.

Disclosure forms provided by the authors are available with the full text and PDF of this article at www.ajnr.org.

\section{REFERENCES}

1. Weimar C, Goertler M, Harms L, et al. Distribution and outcome of symptomatic stenoses and occlusions in patients with acute cerebral ischemia. Arch Neurol 2006;63:1287-91 CrossRef Medline

2. Lall A, Yavagal DR, Bornak A. Chronic total occlusion and spontaneous recanalization of the internal carotid artery: natural history and management strategy. Vascular 2021;29:733-41 CrossRef Medline

3. Hackam DG. Prognosis of asymptomatic carotid artery occlusion: systematic review and meta-analysis. Stroke 2016;47:1253-57 CrossRef Medline

4. Powers WJ, Derdeyn CP, Fritsch SM, et al. Benign prognosis of never-symptomatic carotid occlusion. Neurology 2000;54:878-82 CrossRef Medline

5. Flaherty ML, Flemming KD, McClelland R, et al. Population-based study of symptomatic internal carotid artery occlusion: incidence and long-term follow-up. Stroke 2004;35:e349-52 CrossRef Medline

6. Thanvi B, Robinson T. Complete occlusion of extracranial internal carotid artery: clinical features, pathophysiology, diagnosis and management. Postgrad Med J 2007;83:95-99 CrossRef Medline

7. Gupta A, Chazen JL, Hartman M, et al. Cerebrovascular reserve and stroke risk in patients with carotid stenosis or occlusion: a systematic review and meta-analysis. Stroke 2012;43:2884-91 CrossRef Medline

8. Underhill HR, Hatsukami TS, Cai J, et al. A noninvasive imaging approach to assess plaque severity: the carotid atherosclerosis score. AJNR Am J Neuroradiol 2010;31:1068-75 CrossRef Medline

9. Takaya N, Yuan C, Chu B, et al. Presence of intraplaque hemorrhage stimulates progression of carotid atherosclerotic plaques: a high-resolution magnetic resonance imaging study. Circulation 2005;111:2768-75 CrossRef Medline

10. de Havenon A, Mossa-Basha M, Shah L, et al. High-resolution vessel wall MRI for the evaluation of intracranial atherosclerotic disease. Neuroradiology 2017;59:1193-1202 CrossRef Medline

11. Dieleman N, Yang W, Abrigo JM, et al. Magnetic resonance imaging of plaque morphology, burden, and distribution in patients with symptomatic middle cerebral artery stenosis. Stroke 2016;47:17971802 CrossRef Medline
12. Hui FK, Zhu X, Jones SE, et al. Early experience in high-resolution MRI for large vessel occlusions. J Neurointerv Surg 2015;7:509-16 CrossRef Medline

13. Murata K, Murata N, Chu BC, et al; CARE-II Study Collaborators. Characterization of carotid atherosclerotic plaques using 3-dimensional MERGE magnetic resonance imaging and correlation with stroke risk factors. Stroke 2020;51:475-80 CrossRef Medline

14. Narumi S, Sasaki M, Natori T, et al. Carotid plaque characterization using 3DT1-weighted MR imaging with histopathologic validation: a comparison with 2D technique. AJNR Am J Neuroradiol 2015;36:75156 CrossRef Medline

15. Zhang J, Ding S, Zhao H, et al. Evaluation of chronic carotid artery occlusion by non-contrast 3D-MERGE MR vessel wall imaging: comparison with 3D-TOF-MRA, contrast-enhanced MRA, and DSA. Eur Radiol 2020;30:5805-14 CrossRef Medline

16. Chai S, Sheng Z, Xie W, et al. Assessment of apparent internal carotid tandem occlusion on high-resolution vessel wall imaging: comparison with digital subtraction angiography. AJNR Am J Neuroradiol 2020;41:693-99 CrossRef Medline

17. Zhou J, Cai SF, Yuan XS, et al. Remodeling of occluded internal carotid artery in vessel wall magnetic resonance imaging. Int $J$ Neurosci 2020 Nov 18. [Epub ahead of print] CrossRef Medline

18. Yang Y, Liu X, Wang R, et al. A treatment option for symptomatic chronic complete internal carotid artery occlusion: hybrid surgery. Front Neurosci 2020;14:392 CrossRef Medline

19. Nguyen-Huynh MN, Lev MH, Rordorf G. Spontaneous recanalization of internal carotid artery occlusion. Stroke 2003;34:1032-34 CrossRef Medline

20. Bouthillier A, van Loveren HR, Keller JT. Segments of the internal carotid artery: a new classification. Neurosurgery 1996;38:425-32; discussion 432-33 CrossRef Medline

21. Chen X, Wang J, Liu Y, et al. Proximal internal carotid artery stenosis associates with diffuse wall thickening in petrous arterial segment of Moyamoya disease patients: a three-dimensional magnetic resonance vessel wall imaging study. Neuroradiology 2019;61:29-36 CrossRef Medline

22. Zhao HL, Wang JN, Liu XS, et al. Assessment of carotid artery atherosclerotic disease by using three-dimensional fast black-blood MR imaging: comparison with DSA. Radiology 2015;274:508-16 CrossRef Medline

23. Wang Z, Lu M, Liu W, et al. Assessment of carotid atherosclerotic disease using three-dimensional cardiovascular magnetic resonance vessel wall imaging: comparison with digital subtraction angiography. J Cardiovasc Magn Reson 2020;22:18 CrossRef Medline

24. Paraskevas KI, Mikhailidis DP, Liapis CD. Internal carotid artery occlusion: association with atherosclerotic disease in other arterial beds and vascular risk factors. Angiology 2007;58:329-35 CrossRef Medline

25. Gomori JM, Grossman RI, Hackney DB, et al. Variable appearances of subacute intracranial hematomas on high-field spin-echo MR. AJR Am J Roentgenol 1988;150:171-78 CrossRef Medline

26. Gomori JM, Grossman RI, Goldberg HI, et al. Intracranial hematomas: imaging by high-field MR. Radiology 1985;157:87-93 CrossRef Medline

27. Chu B, Kampschulte A, Ferguson MS, et al. Hemorrhage in the atherosclerotic carotid plaque: a high-resolution MRI study. Stroke 2004;35:1079-84 CrossRef Medline

28. Al-Smadi AS, Abdalla RN, Elmokadem AH, et al. Diagnostic accuracy of high-resolution black-blood $\mathrm{MRI}$ in the evaluation of intracranial large-vessel arterial occlusions. AJNR Am J Neuroradiol 2019;40:954-59 CrossRef Medline

29. Chen $\mathrm{X}, \mathrm{Zhao} \mathrm{H}$, Chen Z, et al. Association between proximal internal carotid artery steno-occlusive disease and diffuse wall thickening in its petrous segment: a magnetic resonance vessel wall imaging study. Neuroradiology 2017;59:485-90 CrossRef Medline 
30. Bo WJ, McKinney WM, Bowden RL. The origin and distribution of vasa vasorum at the bifurcation of the common carotid artery with atherosclerosis. Stroke 1989;20:1484-87 CrossRef Medline

31. Barker SG, Causton BE, Baskerville PA, et al. The vasa vasorum of the rabbit carotid artery. J Anat 1992;180:(Pt 2):225-31 Medline

32. Xie YB, Yang Q, Xie GX, et al. Improved black-blood imaging using DANTE-SPACE for simultaneous carotid and intracranial vessel wall evaluation. Magn Reson Med 2016;75:2286-94 CrossRef Medline
33. Fan ZY, Zuehlsdorff S, Liu X, et al. Prospective self-gating for swallowing motion: A feasibility study in carotid artery wall MRI using three-dimensional variable-flip-angle turbo spin-echo. Magn Reson Med 2012;67:490-98 CrossRef Medline

34. Chen YH, Leong WS, Lin MS, et al. Predictors for successful endovascular intervention in chronic carotid artery total occlusion. JACC Cardiovasc Interv 2016;9:1825-32 CrossRef Medline 